\section{Embedding Brains In Egg Yolk For Cryosectioning}

\author{
Karen Ayyad
}

V.A. Medical Center/Presbyterian Hospital, Dallas TX

Fifteen years ago, I learned from a neurologist how to embed perfused, glutaraldehyde-fixed rat brain in egg yolk (from store-bought, separated chicken eggs). The original technical note by Adoff (reference below) for

\section{DIRECTOR/MANAGER OF OPERATIONS OF THE SCANNING AND TRANSMISSION ELECTRON MICROSCOPY RESEARCH FACILITY}

GENERAL: The Marine Geosciences Division, Naval Research Laboratory (NRL), Stennis Space Center, MS, is seeking to fill the position of Director/Manager of the division's STEM research facility. The successful candidate will be an accomplished transmission electron microscopist with a Ph.D. degree in a closely related academic field, or possibly a Ph.D. equivalent.

FACILITY MANAGEMENT AND OPERATION: The successful candidate will serve as the director and manager of the facility and will be fully responsible for:

- Competent, efficient, economical and safe operation and maintenance of NRL's $300 \mathrm{kV}$ STEM with Environmental Cell and supporting instrumentation for imaging and analyses - including Scanning Mode Microscopy (SEM); Energy Dispersive X-ray Spectroscopy (EDS); Electron Loss Spectroscopy (EELS); a slow scan, charge-coupled device (CCD) camera and other data acquisition systems, and $100 \mathrm{kV}$ Transmission Electron Microscope with Environmental Cell,.

- Staffing the facility with high quality support personnel.

- Setting up operating procedures and protocol for all aspects of work in the facility.

- Supervision and training of technical personnel.

- Overseeing financial operations of the facility.

- Setting up a cataloging/archiving system for samples, imagery, etc.

- Ensuring that operations conform to all NRL/Navy health and safety regulations and standards.

RESEARCH: As the senior electron microscopy expert, the incumbent conducts forefront basic and applied research directed towards developing state-of-the-art microscopy techniques and applying these to investigations of complex problems in marine geomaterials, geochemistry, microbiology and environmental processes. A primary focus will be directed towards Naval problems particularly as involves understanding fundamental processes associated with formation, deposition, and alteration of cohesive marine sediments and the impact on the geoacoustic and geotechnical properties of the sediments and behavior of these sediments in the marine environment. The inclusion of the environmental cell into the STEM provides NRL with one of the unique capabilities in the world. With the STEM and Environmental Cell, the incumbent will be able to conduct forefront research on gaseous and aquecas chemical processes at the molecular scale.

For further information, contact:

Dr. Philip J. Valent (Code 7401)

Naval Research Laboratory

Stennis Space Center, MS 39529-5004

Tel.: 228-688-4650, Fax: 228-688-4093,

eMail: phil.valent@inrlssc.navy.rnil

The Naval Research Laboratory is an Affirmative ActioniEqual Opportunity Employer whole dog brain tissue was used, and we adapted the procedure for rat brain.

1) Fix the whole brain. minus the meninges, in $10 \%$ formalin or glutaraldehydesucrose ( $1 \%$ glutaraldehyde $+30 \%$ sucrose), then place in a specimen container of $30 \%$ sucrose, $10 \%$ formalin and $0.9 \% \mathrm{NaCl}$. The brain is left in this solution until it sinks (less than a week). This step prevents formation of ice crystals when freezing (The meninges must be removed, or the brain will not adhere to the egg yolk.).

2) Make five-sided cubical boats slightly larger than the rat brain (in length, width, and depth) from a pattern drawn on and cut out of index cards. The boats should be large enough to allow a $3 \mathrm{~mm}$ border of egg yolk around the specimen. Tape the four sides of the boat together with Scotch or similar tape. (See illustration.)

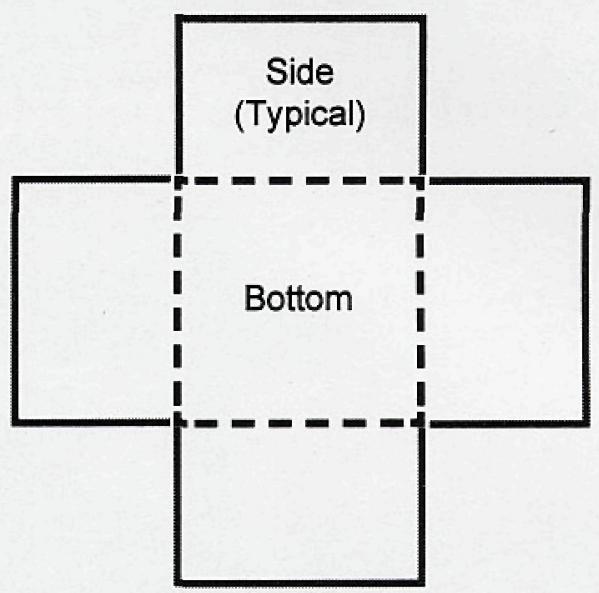

3) The yolks should be separated from the whites just before they are needed and stirred for 5 minutes (we used stir bars and stir plates).

4) The brain is blotted dry before embedding.

5) A thin layer of the stirred egg yolk is poured into the paper boats, the brain is placed in the boats, oriented as desired, and the specimen is covered with the yolk.

6) Suspend the boats over $10 \%$ formalin in a covered container. Adoff did this for 3 weeks at $4^{\circ} \mathrm{C}$. We kept ours at room temperature for much less time, only until the yolks were dry and stiff. A raised platform can be used. The idea is to keep the specimen boat from being submerged in the formalin.

7) Remove the paper boat from the jar and carefully pull off the boat from the yolk-embedded brain.

8) Mount the yolk-embedded brain on a freezing microtome (a cryostat would be even better) with OCT compound, orienting as desired.

We froze the block for secfloning with finely crushed dry ice and used a sliding microtome attached to the bench top (with $\mathrm{CO}_{2}$ line attachment). A Vibratome would work, however the freezing helped give the specimen an added stiffness.

We cut very thick sections of $50 \mu \mathrm{m}$, which could be floated in water or placed directly into incubation media and later mounted on egg albumin-coated, subbed slides for further staining or processing.

The rationale behind the yolk embedding and frozen sectioning of the fixed tissue was that we could very easily control sectioning to readily obtain either sagittal, transverse, or coronal sections orientation by mounting any of the five flat, solid specimen block surfaces. During sectioning, it was very easy to see the brain architectonics so that we knew at what level we were, how far to trim down, and which sections to save.

Adoff states "The primary function of the egg yolk is support. The egg yolk adheres to and remains with the tissue throughout cutting, mounting. and staining... is especially helpful in handling the sections."

Adoff, L.M. 1981. Egg Yolk Embedding for Frozen Whole Brain Sections, Stain Technology 56(2): 125-126 in the Notes on Technic section. 


\section{Keeping a good image around the lab just got a lot easier. Quartz PCI.}

With the Windows-based Quartz $\mathrm{PCI}^{\mathrm{TM}}$ system, you can capture and manage highresolution digital images from just about any image-producing device. And do so with a single - and familiar - family of software that lets you smoothly work with SEMs, STEMs, TEMs, light microscopes, video sources, TWAIN devices and CD SEMs. You end continuous learning of new software. Easily network diverse instruments. And conveniently enter all types of images into one database.

Further, after acquiring an image, PCI's processing and display capabilities let you do just about anything you want with it-from annotating, zooming, smoothing and sharpening it to making gamma adjustments and constructing anaglyph stereo images. And you're free to add your own custom functions as well. Now, think about your database. PCl's scalable family of database solutions ensures your images - plus related documents, custom fields and tables - become part of a powerful database that provides data when,

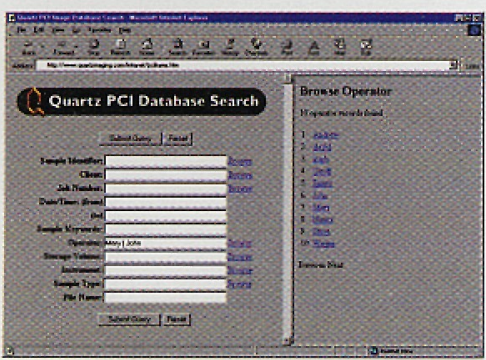

The PCI Intranet Image Server lets you use your Web browser to search for images. where, and in the form needed. OBDC compliance ensures compatibility with third-party tools like Microsoft ${ }^{\circ}$ Access ${ }^{\text {TM }}$ And PCI's Workgroup Database, for facilities with up to 25 users, is upgradable to our Enterprise Database which - based on either Oracle, ${ }^{\oplus}$ or Microsoft ${ }^{\oplus}$ SQL Server ${ }^{\text {TM }}$ supports thousands. With our Intranet Image Server, you can perform queries and retrieve images via standard Web browsers. And, for off-line storage, there's built-in support for removable media such as CD-ROMs or magneto-optical disks.

So. If easily acquiring, analyzing, manipulating, annotating, transmitting, archiving and retrieving high-resolution images from various sources is in your future, you'll want Quartz PCI details in your hands. You can arrange that easily, too. Visit the Nissei Sangyo Web site for a demonstration, or let us hear from you by phone, FAX or E-mail.

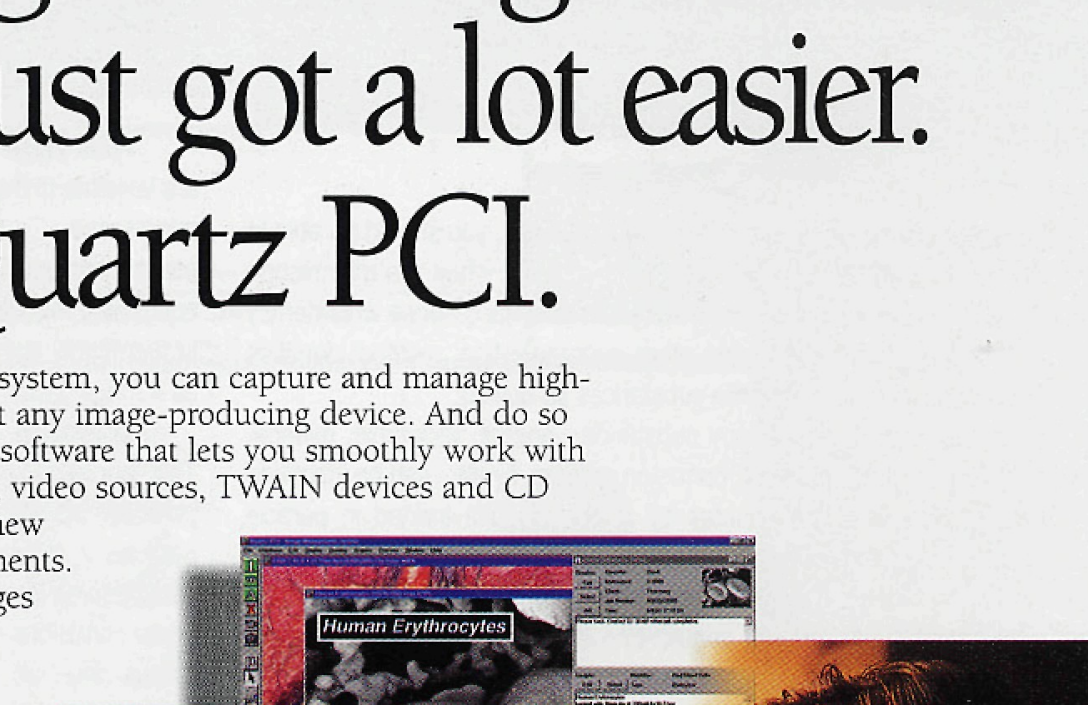

